\title{
Spatial Distribution of Io's Volcanic Activity from Near-IR Adaptive Optics Observations on 100 Nights in 2013-2015
}

\author{
Katherine de Kleer ${ }^{\mathrm{a}}$, Imke de Pater ${ }^{\mathrm{a}, \mathrm{b}, \mathrm{c}}$ \\ ${ }^{a}$ Astronomy Department, University of California, Berkeley, CA 94720 \\ ${ }^{b}$ Delft Institute of Earth Observation and Space Systems, Delft University of Technology, NL-2629 HS Delft, The Netherlands \\ ${ }^{c}$ SRON Netherlands Institute for Space Research 3584 CA Utrecht, The Netherlands
}

Keywords: Io, Volcanism, Infrared Observations, Adaptive Optics, Spectroscopy

Katherine de Kleer

501 Campbell Hall \#3411

University of California at Berkeley

Berkeley, CA 94720

E-mail: kdekleer@berkeley.edu

(C) 2016. This manuscript version is made available under the Elsevier user license http://www.elsevier.com/open-access/userlicense/1.0/ 


\section{Abstract}

The extreme and time-variable volcanic activity on Jupiter's moon Io is the result of periodic tidal forcing. The spatial distribution of Io's surface heat flux provides an important constraint on models for tidal heat dissipation, yielding information on interior properties and on the depth at which the tidal heat is primarily dissipated. We analyze the spatial distribution of 48 hot spots based on more than 400 total hot spot detections in adaptive optics images taken on 100 nights in 2013-2015 (data presented in de Kleer and de Pater, 2016). We present full surface maps of Io at multiple near-infrared wavelengths for three epochs during this time period, and show that the longitudinal distribution of hot spots has not changed significantly since the Galileo mission.

We find that hot spots that are persistently active at moderate intensities tend to occur at different latitudes/longitudes than those that exhibit sudden brightening events characterized by high peak intensities and subsequent decay phases. While persistent hot spots are located primarily between $\pm 30^{\circ} \mathrm{N}$, hot spots exhibiting bright eruption events occur primarily between $40^{\circ}-65^{\circ}$ in both the northern and southern hemispheres. In addition, while persistent hot spots occur preferentially on the leading hemisphere, all bright eruptions were detected on the trailing hemisphere, despite the comparable longitudinal coverage of our observations to both hemispheres. A subset of the bright hot spots which are not intense enough to qualify as outburst eruptions resemble outbursts in terms of temporal evolution and spatial distribution, and may be outbursts whose peak emission went unobserved, or else scaled-down versions of the same phenomenon. A statistical analysis finds that large eruptions are more spatially clustered and occur at higher latitudes than $95 \%$ of simulated datasets that assume that eruptions occur at random and independent locations.

The preferential occurrence of bright, violent eruptions at higher latitudes supports the idea that a deeper magma source supplies these events, as has been previously hypothesized. The monotonic eastward progression of bright eruptions at southern latitudes from $300^{\circ}$ to $200^{\circ} \mathrm{W}$ also suggests a possible eruption triggering mechanism operating across distances of $\sim 500 \mathrm{~km}$. A comparison to tidal heating models finds a good correspondence between recent models incorporating a partially-fluid interior (Tyler et al., 2015) and hot spots in the leading hemisphere as well as persistent hot spots. However, hot spots on the trailing hemisphere and bright eruptions do not match these models well, corresponding better to standard deepmantle heating models (Segatz et al., 1988), although this match is still imperfect.

\section{Introduction}

An understanding of the interior properties and geophysics of rocky bodies in the Solar System is crucial for providing a framework in which to interpret observations of extrasolar planets. In cases of tidally-heated 
bodies with subsurface oceans such as Europa and Enceladus, models for how and where tidal heat is dissipated in planetary interiors are needed to answer questions about the presence and history of liquid water, which affects their long-term habitability. Such models would also help predict whether extrasolar planets might have volcanism, global magma layers, or even liquid water. However, a major challenge to understanding planetary interiors is the sparsity of information that can be obtained remotely, particularly without in situ gravity and magnetometer measurements.

Io represents a unique case where an interior process (tidal heating) manifests on the surface in a clearly detectable way, as extreme volcanic activity. This moon therefore provides a unique testbed for interior processes, tidal heat dissipation in particular. The distribution of heat flow on a planet's surface puts an important constraint on models of tidal heat dissipation, and in particular on how deep within the interior the heat is dissipated. Models show that if the heat is primarily dissipated in the deep mantle, Io's surface heat flux should be highest at the poles, and should be higher on the leading/trailing hemispheres (longitudes of $90^{\circ}$ and $270^{\circ} \mathrm{W}$ ) than on the sub- and anti-jovian hemispheres (longitudes of $0^{\circ}$ and $180^{\circ} \mathrm{W}$ ). In contrast, if the heat is primarily dissipated in a fluid asthenosphere, surface heat flux should be very low at the poles, reaching maxima around the sub- and anti-jovian points at $\pm 30^{\circ}$ latitude, with secondary maxima at the equatorial leading and trailing points (Segatz et al., 1988; Ross et al., 1990). Io's true heat dissipation is likely a combination of these idealized scenarios, potentially with lateral convective flows that smooth out the surface heat flux pattern (Hamilton et al., 2013; Tyler et al., 2015; Davies et al., 2015).

Much recent progress has been made in characterizing the distribution of volcanic heat flow on Io, and on developing realistic tidal heating models that can be tested against observations. Previous work has assumed that the volcanic heat flow distribution on the surface can be used as a tracer of the distribution of internal heat flow. We follow this assumption, while noting that the connection between interior heating and its surface expression as volcanism has not been checked, and these properties may correlate imperfectly.

Early results from the Galileo mission found that there is a lower concentration of volcanic centers at high latitudes (Carr et al. 1998; Schenk et al. 2001), but that high latitude paterae are larger, suggesting a different eruption character (Radebaugh et al., 2001). Schenk et al. (2001) and Radebaugh et al. (2001) also noted a bimodal distribution of volcanic centers that peaks near $330^{\circ} \mathrm{W}$ and $150^{\circ} \mathrm{W}$. Lopes et al. (2004) analyzed 166 volcanic centers observed by Galileo NIMS (78 at the highest resolution), and confirm that the distribution of active centers also has a peak at $150^{\circ} \mathrm{W}$. In a series of recent papers, Veeder et al. (2009; 2011; 2012; 2015) identified additional faint emission sources in the NIMS dataset and characterized the thermal emission from $\sim 250$ total volcanic centers observed by Galileo. A heat flow map based on this analysis is presented in Davies et al. (2015), and indicates a non-uniform distribution of heat flow which is not a good match to any of the tidal heating model end-members. These authors note a lack of close 
correlation between density of volcanic centers and local heat flow. The clustering of hot spots and paterae on Io, as identified from the first global geologic map of Io (Williams et al., 2011) as well as from space- and ground-based observations, shows more uniformity and less randomness in equatorial regions compared to the poles. This suggests that volcanic centers are independently formed at high latitudes but that magma scavenging occurs in the near-equatorial region (Hamilton et al., 2013), consistent with a shallow magma source near the equator and a deeper source for eruptions at high latitudes.

All of the above authors identify an eastward offset of $30-60^{\circ}$ in the concentration of volcanic activity from the locations predicted by heating models. In addition, the longitudes of the maxima based on active volcanism fall $\sim 30^{\circ}$ eastwards of the maxima based on paterae (Hamilton et al., 2013). In the first model to incorporate the fluid effects of a partially-molten interior layer, Tyler et al. (2015) demonstrate that the longitudinal offset can be explained by including the tidal response of a fluid layer such as the "magma ocean" implied by constraints derived from the Galileo magnetometer data (Khurana et al., 2011). They propose that high-latitude eruptions may be driven by deep-mantle heating, while equatorial volcanoes may have a shallow magma source. Such a scenario would imply hotter, more violent polar volcanoes occurring at random locations, while equatorial volcanoes may be cooler, less violent, and distributed more evenly due to competition for shallow magma reservoirs (Hamilton et al., 2013)

In a companion paper (de Kleer and de Pater, 2016), we present a new dataset of ground-based adaptive optics images of Io in the near-infrared, including 409 detections of activity from at least 48 distinct hot spots over a period from Aug 2013 through Dec 2015. The high cadence of our observations samples both transient and long-lived eruptions, and the number of detections is large enough to provide a statistical sample for an analysis of the spatial distribution of active centers. While the time-variability of hot spot emission is discussed in de Kleer and de Pater (2016), this article focuses on the spatial distribution of the detected hot spots. In Section 2 we summarize our observations. The spatial distribution of volcanic activity is presented in Section 3, including near-infrared surface maps for multiple epochs and wavelengths. Section 4 presents a statistical analysis of the distribution of large eruptions, and Section 5 discusses the implications of our results, including a comparison with patera and hot spot distributions determined by past work and an investigation of correlations between eruption characteristics and hot spot locations. Our main conclusions are summarized in Section 6.

\section{Observations and Data Processing}

The analysis in this paper is based on near-infrared $(1-5 \mu \mathrm{m})$ images of Io obtained on 100 nights between August 2013 and December 2015 with adaptive optics at the Gemini N and Keck telescopes. The 
observations, data reduction, and analysis methods are described in a companion paper (de Kleer and de Pater, 2016), and the hot spot positions and intensities are listed in Table B.4 of that article. The reader is also referred to that paper for a detailed study of the uncertainties on the retrieved hot spot properties.

\subsection{Projection and Surface Maps}

The Keck data are projected with an equirectangular projection onto a grid of constant latitude and longitude intervals. Before projection, the images are deconvolved using the stellar point spread function (PSF) for each night of observation using the AIDA routine (Hom et al., 2007), in order to sharpen the images and enhance contrast. All images are corrected for limb darkening according to a Minnaert law:

$$
I=I_{0} \mu_{0}^{k} \mu^{k-1}
$$

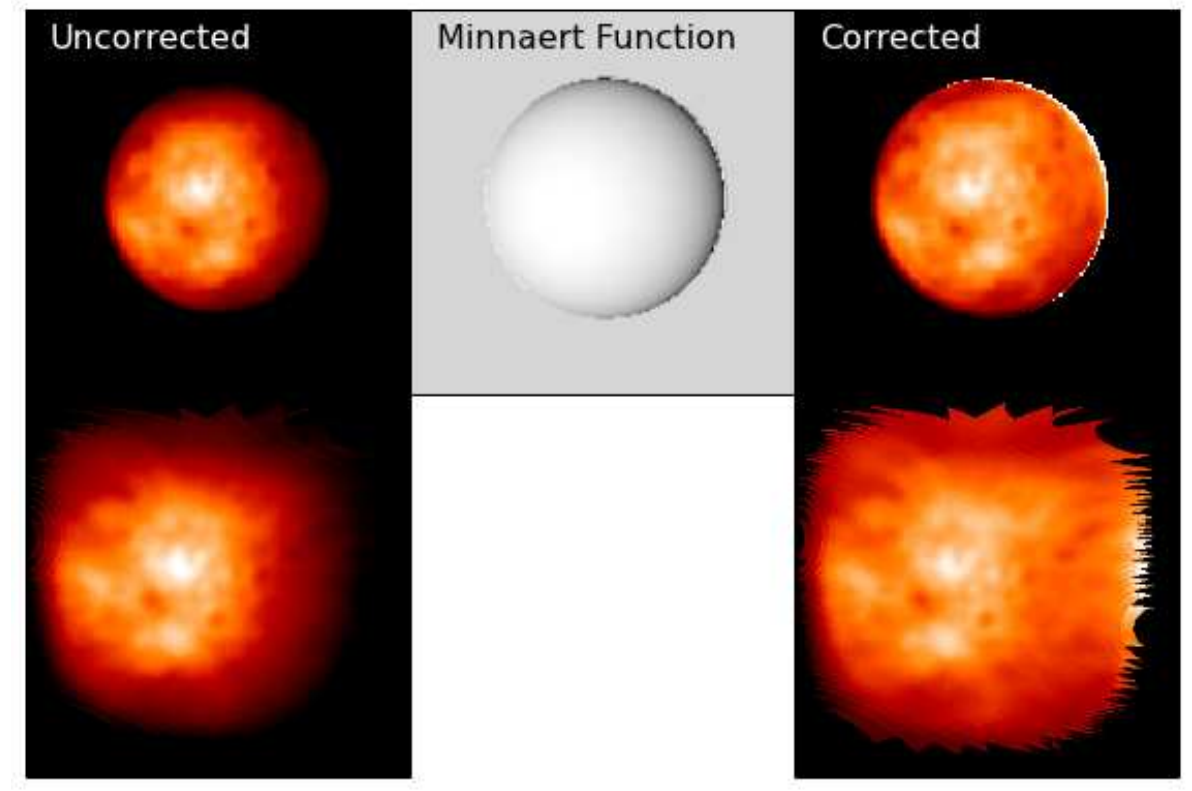

Figure 1: Demonstration of limb darkening correction: Keck Kc-band data from Oct 30, 2014, before and after limb-darkening correction by a Minnaert law with $k=0.67$. The top row shows the images before and after the correction, and bottom row shows the same two images after projection onto a regular latitude-longitude grid.

\footnotetext{
${ }^{1}$ Ephemeris from JPL HORIZONS System: ssd.jpl.nasa.gov/horizons.cgi
} 
The projected images within each of three observing epochs are stitched together to produce global maps in each filter. At longitudes which were observed on multiple nights, the map is the median-average of all available observations. Each observation is scaled so that the over-lapping regions on all nights match as well as possible, using the highest-quality image as a photometric reference. After applying the flux-calibration procedures described in de Kleer and de Pater (2016), we typically need to scale the projected images by a few percent at most.

\section{Results}

\subsection{Multi-Wavelength Surface Maps}

Maps of Io's surface at near-infrared wavelengths provide an informative visualization of the distribution, magnitude, and temperature of activity on Io. We present surface maps for three distinct periods in Figure 2, in five near-infrared filters (K-cont, L', Br- $\alpha$, Br- $\alpha$-cont, Ms; wavelengths of 2.27, 3.78, 3.99, 4.05, and 4.67 $\mu \mathrm{m})$, based on Keck observations. For the last time period, we present additional maps in narrowband filters at 3.06 and $3.29 \mu \mathrm{m}$. At $2.27 \mu \mathrm{m}$, the intensity is almost entirely due to reflected sunlight, and the maps show the near-infrared albedo distribution. At longer wavelengths, thermal emission from active volcanic regions is an increasingly significant component of the emission. At intermediate wavelengths $(\sim 3 \mu \mathrm{m})$, the thermal emission and albedo distribution can both be seen, and the location of emission within large dark paterae such as Loki Patera and Pele is immediately apparent.

Io's surface is covered in $\mathrm{SO}_{2}$ frost, which has a broad absorption band centered near $4 \mu \mathrm{m}$. The filter at $4.05 \mu \mathrm{m}(\mathrm{Br}-\alpha)$ falls within this absorption band, while the filter at $3.99 \mu \mathrm{m}(\mathrm{Br}-\alpha$-cont) lies just outside it and can be considered the nearby continuum. As can be seen in Figure 2, the absorption of incident sunlight within this band increases the contrast of hot spots to the surface, particularly in regions of greater $\mathrm{SO}_{2}$ frost, i.e. the brighter regions at other wavelengths.

\subsection{Spatial Distribution of Volcanic Activity}

The high spatial resolution of our observations allows us to constrain the location of hot spots on Io's surface to within a few degrees or better in latitude and longitude. We present the position of each hot spot detection on Figure 3, where each shaded circle represents a single detection, and the size of the circle indicates the magnitude of the 3.8- $\mu \mathrm{m}$ intensity. An animation is also included in the Supplementary Material which steps through time plotting each of the detections shown in Figure 3 at its time of occurrence. In Section 5.1 we analyze this distribution in light of observational biases to make an estimate of the true longitudinal distribution of volcanic activity on Io in 2013-2015, which we compare with previous distributions and with the predictions of tidal heat dissipation models. 

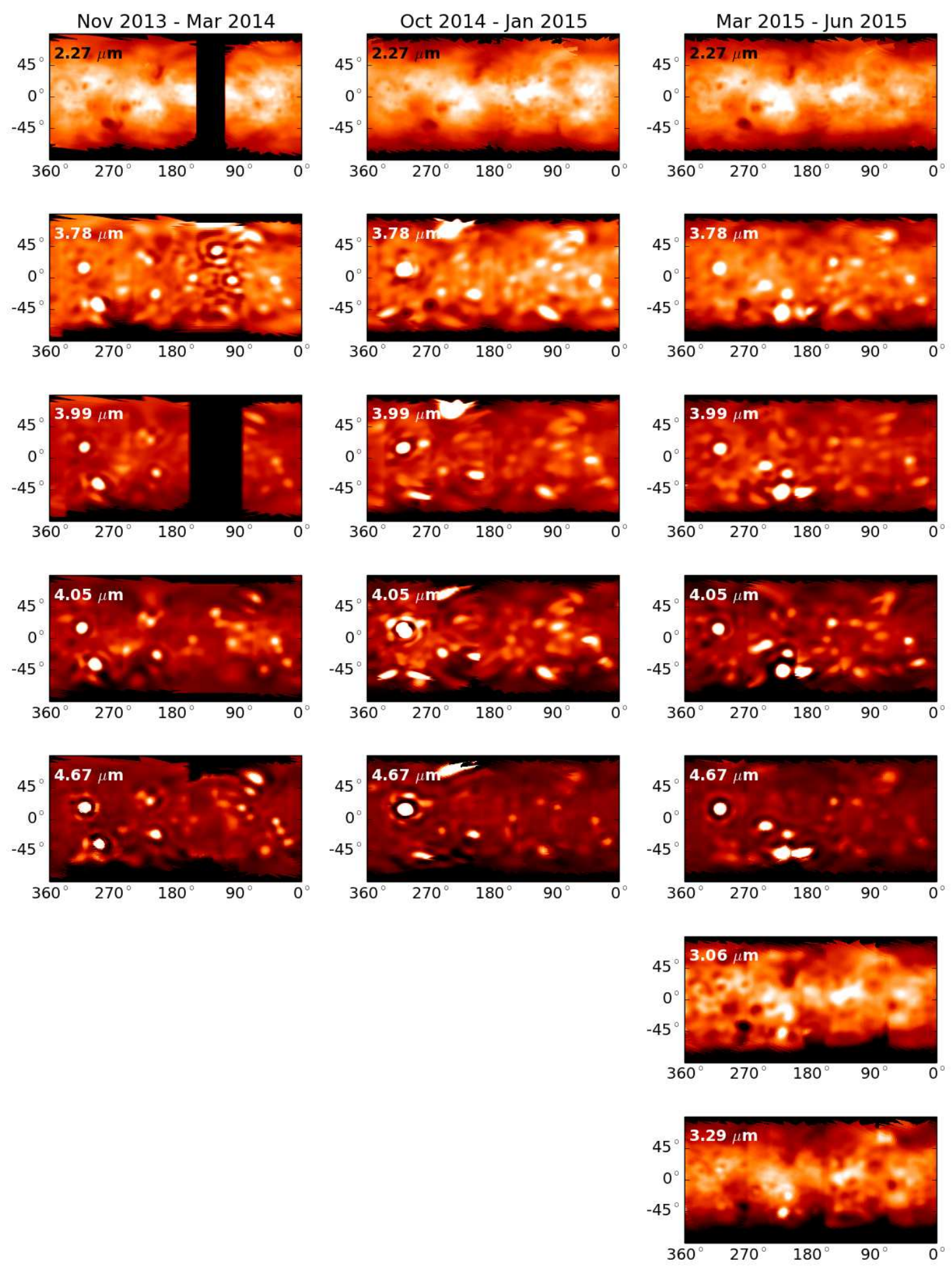

Figure 2: Surface maps of Io at multiple near-infrared wavelengths. Each map is made up of several separate observations with the Keck telescope, projected onto a rectangular grid and stitched together. Latitude and longitude are labeled in ${ }^{\circ} \mathrm{N}$ and ${ }^{\circ} \mathrm{W}$.

\section{4. Spatial Clustering of Large Eruptions}

The most striking feature of Figure 3 is the asymmetric distribution of the locations of the largest 3 eruptions, which are detected exclusively between $\sim 200$ and $\sim 300^{\circ} \mathrm{W}$, and often at mid to high latitudes. 


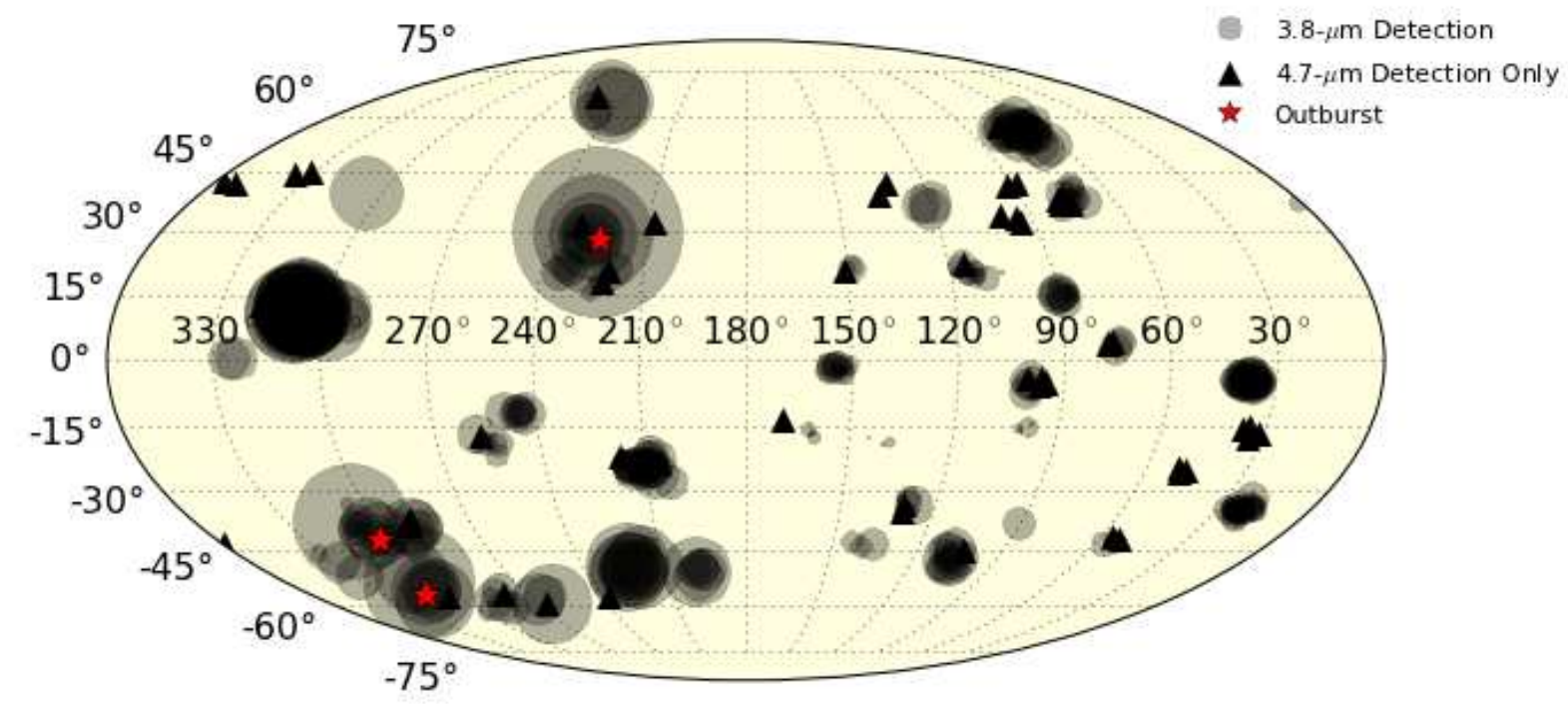

Figure 3: All hot spot detections from August 2013 through December 2015, shown with a Mollweide equal-area projection. Each circle represents a $3.8-\mu \mathrm{m}$ hot spot detection; the size of the circle corresponds logarithmically to the $3.8-\mu \mathrm{m}$ intensity, and darker regions are where a hot spot was detected multiple times. Hot spot detections at $4.7 \mu \mathrm{m}$ correspond to lower-temperature events. The timing of each detection shown here can be seen visually in the animation included in the Supplementary Material.

1 We test the significance of this apparent clustering of eruptions by seeking a quantitative answer to the question: what is the probability that the locations of large eruptions are random, given the observations?

We restrict this analysis to sites that hosted large eruptions, which we define as a peak L'-band intensity exceeding $30 \mathrm{GW} / \mu \mathrm{m} / \mathrm{sr}$. We include both Loki Patera and outburst eruptions in this classification; the nine hot spots meeting this intensity cut-off are given in Table 1. The three brightest detections which did not meet this cut-off are also listed, and are used to test the robustness of our analysis to intensity cut-off.

We note that this cut-off ensures that our dataset is complete; our detection limit analysis indicates that we detect all eruptions above this flux level, at both telescopes, and at all disk locations (de Kleer and de Pater, 2016). All detections in Table 1 share the distinguishing feature of rapid brightening events followed by a decay over a timescale of days to weeks (or months in the case of Loki Patera), implying that these events constitute a physically-distinct class.

We test the null hypothesis that large eruptions occur in random locations as follows. We create a sample of 100,000 simulated datasets, each of which contains nine eruptions at random and independent locations on Io's surface, matching the number of bright detected hot spots in our dataset. The eruptions are each placed in a box of size $5^{\circ} \times 5^{\circ}$, where the probability of an eruption occurring in a given box is weighted by the surface area contained in that box, and by the fraction of our observations that were sensitive to its longitude. The former weighting factor corrects for the fact that a $5^{\circ} \times 5^{\circ}$ region at high latitudes contains a much smaller area than one near the equator. The map of eruption probabilities, corrected for longitudinal coverage of our observations, is shown in Figure 4. 
Table 1: Brightest eruptions detected with Gemini N/Keck in 2013-2015

\begin{tabular}{lllll}
\hline Site & $\begin{array}{l}\text { Latitude }^{a} \\
{\left[{ }^{\circ} \mathrm{N}\right]}\end{array}$ & $\begin{array}{l}\text { Longitude }^{a} \\
{\left[{ }^{\circ} \mathrm{W}\right]}\end{array}$ & Date $^{b}$ & $\begin{array}{l}\text { Peak 3.8- } \mu \mathrm{m} \text { Intensity } \\
{[\mathrm{GW} / \mu \mathrm{m} / \mathrm{sr}]}\end{array}$ \\
\hline 201308C $\mathrm{C}^{c}$ & $29.0 \pm 1.0$ & $229.8 \pm 2.5$ & $08-29-13$ & $>500$ \\
Rarog Patera $^{c}$ & $-38.7 \pm 2.0$ & $306.0 \pm 2.6$ & $08-15-13$ & $320 \pm 80$ \\
Heno Patera $^{c}$ & $-56.0 \pm 1.3$ & $307.2 \pm 1.8$ & $08-15-13$ & $270 \pm 70$ \\
Loki Patera $^{c}$ & $12.0 \pm 1.0$ & $308.2 \pm 4.2$ & $08-22-13$ & $136 \pm 20$ \\
Kurdalagon Patera $_{\text {Chors Patera }}$ & $-48.8 \pm 1.4$ & $221.3 \pm 2.8$ & $01-26-15$ & $68 \pm 11$ \\
Mithra Patera & $64.8 \pm 1.2$ & $246.7 \pm 3.7$ & $10-22-14$ & $57 \pm 19$ \\
Amaterasu Patera & $-58.1 \pm 0.9$ & $266.2 \pm 2.4$ & $01-10-15$ & $55 \pm 12$ \\
Sethlaus/Gabija Paterae & $39.4 \pm 0.8$ & $304.3 \pm 1.1$ & $12-25-15$ & $43 \pm 6$ \\
\hline PV59 & $-50.0 \pm 0.8$ & $192 \pm 1.2$ & $04-01-15$ & $32.6 \pm 4.9$ \\
P170 & $-38.4 \pm 1.5$ & $290.0 \pm 2.4$ & $02-10-14$ & $17.6 \pm 2.6$ \\
P197 & $-47.8 \pm 1.6$ & $327.9 \pm 1.8$ & $12-02-14$ & $11.7 \pm 1.7$ \\
\hline
\end{tabular}

${ }^{a}$ Latitude and Longitude are the median of all observations from 2013-2015, from de Kleer and de Pater (2016).

${ }^{b}$ Date of peak $3.8-\mu \mathrm{m}$ intensity.

${ }^{c}$ 201308C outburst intensity from de Kleer et al. (2014); Rarog and Heno Paterae outbursts and Loki Patera on 08-22-13 from de Pater et al. (2014).

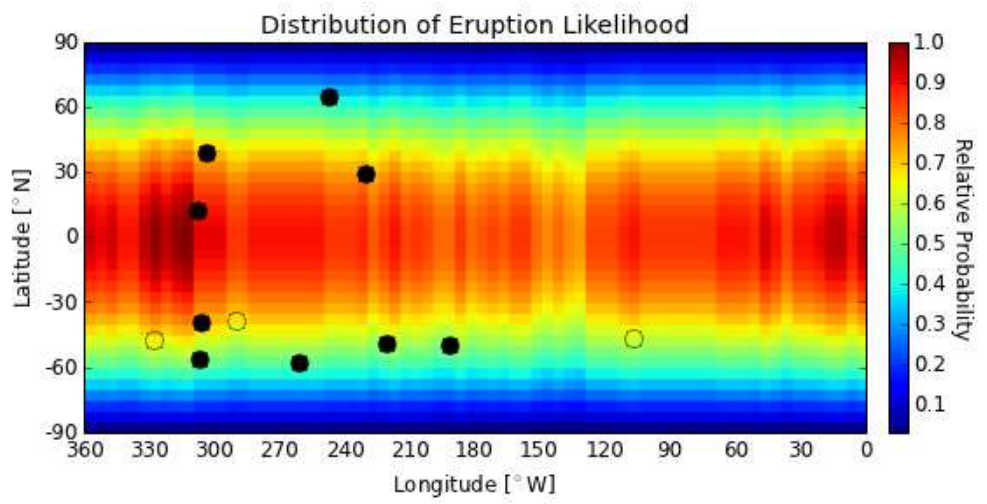

Figure 4: Map of the likelihood of our program detecting a bright eruption in a given latitude-location box, if eruptions are randomly distributed. Map is based on the frequency of observation of each longitude assuming we are sensitive to bright eruptions at all emission angles, and on the surface area in a given latitude-longitude bin. Red regions have a higher eruption likelihood, and blue regions a lower likelihood. Circles indicate observed eruptions: filled circles are the observed large eruptions $(3.8-\mu \mathrm{m}$ flux $>30 \mathrm{GW} / \mu \mathrm{m} / \mathrm{sr})$ used in the analysis, and open circles are the locations of the three brightest hot spot detections that fall below this cut-off, included to demonstrate that the observed clustering and high-latitude locations are not the result of the chosen intensity cut-off.

We then define two metrics to evaluate the spatial distribution of eruptions:

(1) The mean pairwise spacing (in distance on the surface) between eruptions, describing how clustered the set of eruptions is; and

(2) The mean absolute latitude of eruptions, describing whether large eruptions are weighted towards specific latitude ranges. The expected mean absolute latitude for randomly-spaced eruptions is the area- 
1 weighted mean of the absolute latitude $\varphi$ :

$$
\bar{\varphi}=\frac{\int_{0}^{\pi / 2} \varphi \cos (\varphi) d \varphi}{\int_{0}^{\pi / 2} \cos (\varphi) d \varphi}=\frac{\pi}{2}-1 \approx 32.7^{\circ}
$$

Figure 5: Comparison of two hot spot distribution metrics for simulated vs. observed data. Unless otherwise noted, both simulated and observed values are for hot spots where high-intensity $(>30 \mathrm{GW} / \mu \mathrm{m} / \mathrm{sr})$ activity was detected, corresponding to the nine sites listed in Table 1. The comparison indicates that bright hot spots in our dataset are highly clustered and at high latitudes compared to simulations which assume random and independent eruption locations.
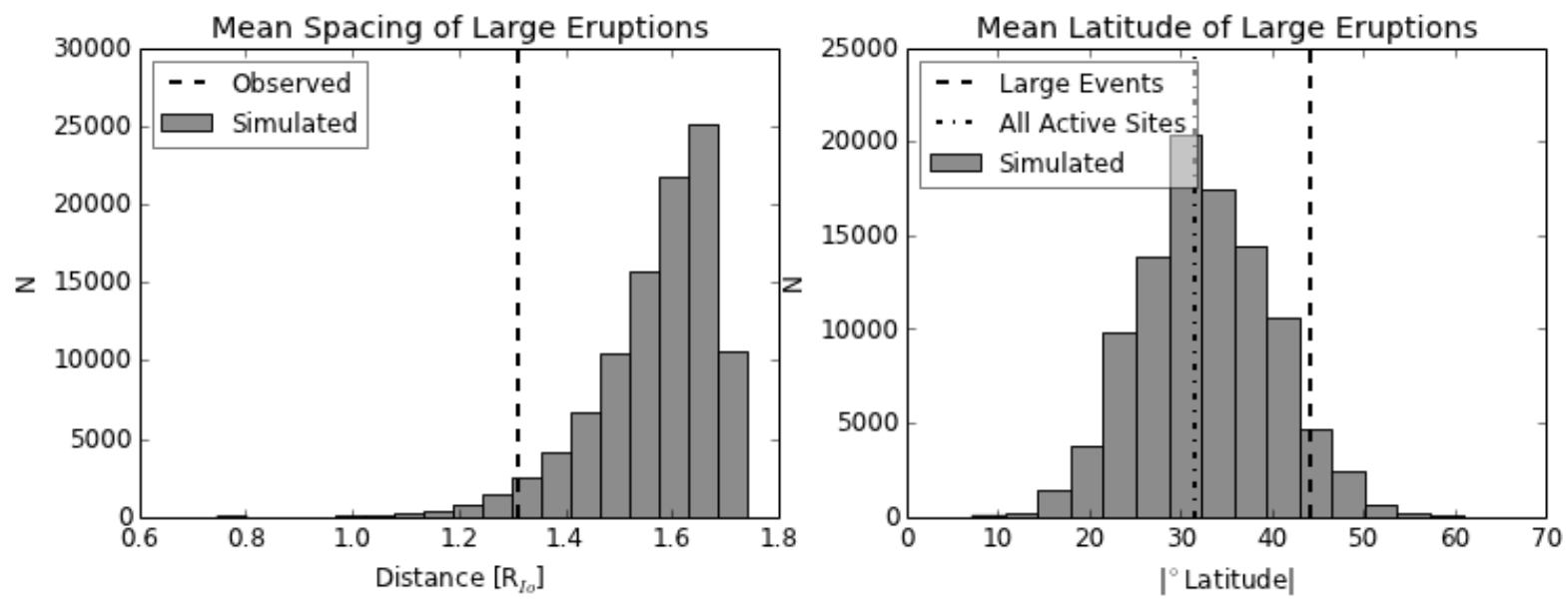

where the cosine term describes the area covered by a fixed latitude interval as a function of $\varphi$.

Figure 5 shows the distribution of these metrics in our simulated data compared to the observations. We find that our dataset has a smaller mean eruption separation than $97 \%$ of the simulated datasets, and a higher mean latitude than $94 \%$ of the simulations. While the mean latitude of the set of large eruptions in a typical simulation is $30-35^{\circ}$, the mean value from our observations is $44^{\circ}$. In contrast, the average latitude of all the sites where we detected activity is $31.5^{\circ}$, which is very close to the expected mean latitude in the case of randomly-distributed eruptions. We note that this number is not corrected for observational biases, and the detection of faint activity at high latitudes is challenging; this correction would shift the mean latitude of all eruptions towards higher latitudes, though it does not affect our analysis of bright eruptions. 


\subsection{Temporal Clustering of Large Eruptions}

The timeline of volcanic activity on Io presented in de Kleer and de Pater (2016) also shows an apparent correlation of large eruptions in time. That is, there is an apparent dichotomy in the timeline, where there are either multiple bright eruptions ongoing and none at all; it is less common to have only a single ongoing bright eruption. We perform a similar analysis to the above to analyze the significance of the temporal correlation. Simulated datasets in the temporal case consist of an array of dates with a 0 or 1 assigned to each date between the first and last day of the program (660 days), representing whether a large eruption occurs. Each dataset is then masked by the dates we actually observed, which selects out only the eruptions we would have detected with our program.

The selected simulated datasets are then evaluated for clustering by dividing them into windows of 10-60 days, and determining how many windows contained new eruptions. In a highly-clustered dataset, all the eruptions would fall in just a few of the windows, while an evenly-distributed dataset might find at most 1-2 eruptions per window. For a given window size, we repeat this procedure with the start date shifted by different numbers of days, to ensure that we are not biased by the exact dates at which the boundaries between successive windows fall.

We find some indication of temporal clustering, but are unable to draw any definitive conclusions. While most choices for window size find that the observed data is more clustered than $80-99 \%$ of the simulated datasets, some choices of window sizes find the clustering significance as low as $55 \%$. In addition, the results are highly sensitive to changing the window size by a few days, shifting the start date, or removing single data points. We therefore conclude that our eruption number is too small to draw conclusions about the temporal correlation of eruptions by this analysis technique.

\section{Discussion}

\subsection{Spatial Distribution of volcanic activity}

During the time period of observation, we find that Io's volcanic activity is non-uniform, exhibiting substantial asymmetries between the leading $\left(0-180^{\circ} \mathrm{W}\right)$ and trailing $\left(180-360^{\circ} \mathrm{W}\right)$ hemispheres, and high vs. low latitudes (see Figure 3). In de Kleer and de Pater (2016), we classify the detected hot spots into two observationally-distinct classes: (1) hot spots with sudden brightening events and high time variability; and (2) persistent hot spots, consistently active for $>1$ year and never exceeding $15 \mathrm{GW} / \mu \mathrm{m} / \mathrm{sr}$ in L' intensity. Figure 6 shows the distribution of volcanic activity, color-coded to indicate which category each eruption belongs to. In addition to the preponderance of bright eruption events in the trailing hemisphere and at midto-high latitudes, we find that the majority of persistent hot spots are located in the leading hemisphere and 
at low latitudes. While detections of lower-intensity hot spots are somewhat biased towards lower latitudes where geometric foreshortening diminishes their intensities by a smaller amount, the hot spots classified as persistent were all of sufficient intensity to be detected at higher latitudes as well. If our analysis did miss persistent hot spots at high latitudes due to observational biases, these hot spots would have been of a lower typical intensity than those at more equatorial latitudes.

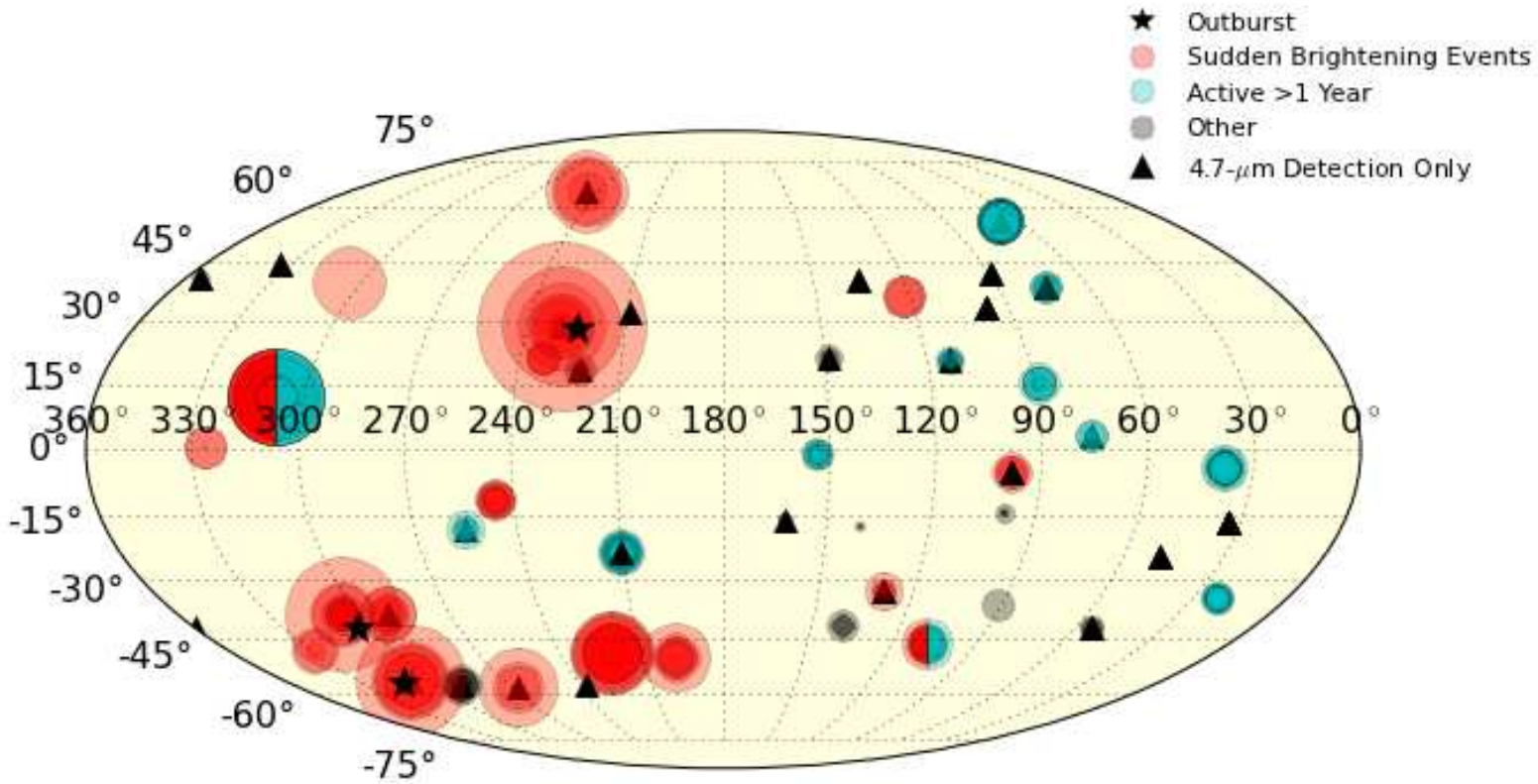

Figure 6: All hot spot detections in 2013-2015. Figure follows Figure 3, except that all detections attributed to the same hot spot have been over-plotted at their median location. Red and cyan coloring indicate eruption classification; points that share both colors exhibit characteristics of both categories, and gray points were detected too few times to classify.

The correlation between persistent hot spots occurring at lower latitudes and short-lived, violent eruptions at high latitudes has been discussed by many previous authors (e.g. Lopes-Gautier et al., 1999; Geissler, 2004). In addition, it has been noted that paterae at high latitudes are less numerous and larger (Schenk et al., 2001; Radebaugh et al., 2001). The high cadence of our observations, which allows us to constrain eruption durations to within weeks or months, has permitted quantitative definitions for these two categories, and a confirmation of this previously-identified correlation.

Of the 48 hot spots detected, only one is located definitively polewards of $60^{\circ}$ (Chors Patera), while five others are located on or within a few degrees of $60^{\circ}$ (Pyerun Patera, Mithra Patera, Chalybes Regio, N Lerna Regio, and Heno Patera). We did not detect any event higher in latitude than $65^{\circ}$ in either hemisphere. If eruptions are randomly distributed on Io's surface, we would expect to see $\sim 9 \%$ of eruptions at higher latitudes than $65^{\circ}$ based on surface area coverage alone, i.e. $\sim 4$ eruptions in our dataset. The lack of detections at these latitudes is due in part to observational biases which prevent detection of faint activity at these latitudes; however, as demonstrated by Figure 7, we still have good sensitivity to eruptions of moderate 
1 brightness up to $\pm 75^{\circ}$.

Figure 7: Maps of the relative sensitivity of our dataset to hot spots of different L'-band intensities, based on the Ionian longitudes sampled by our observations, and the detectability regimes described in de Kleer and de Pater (2016). All three plots follow the same scale.
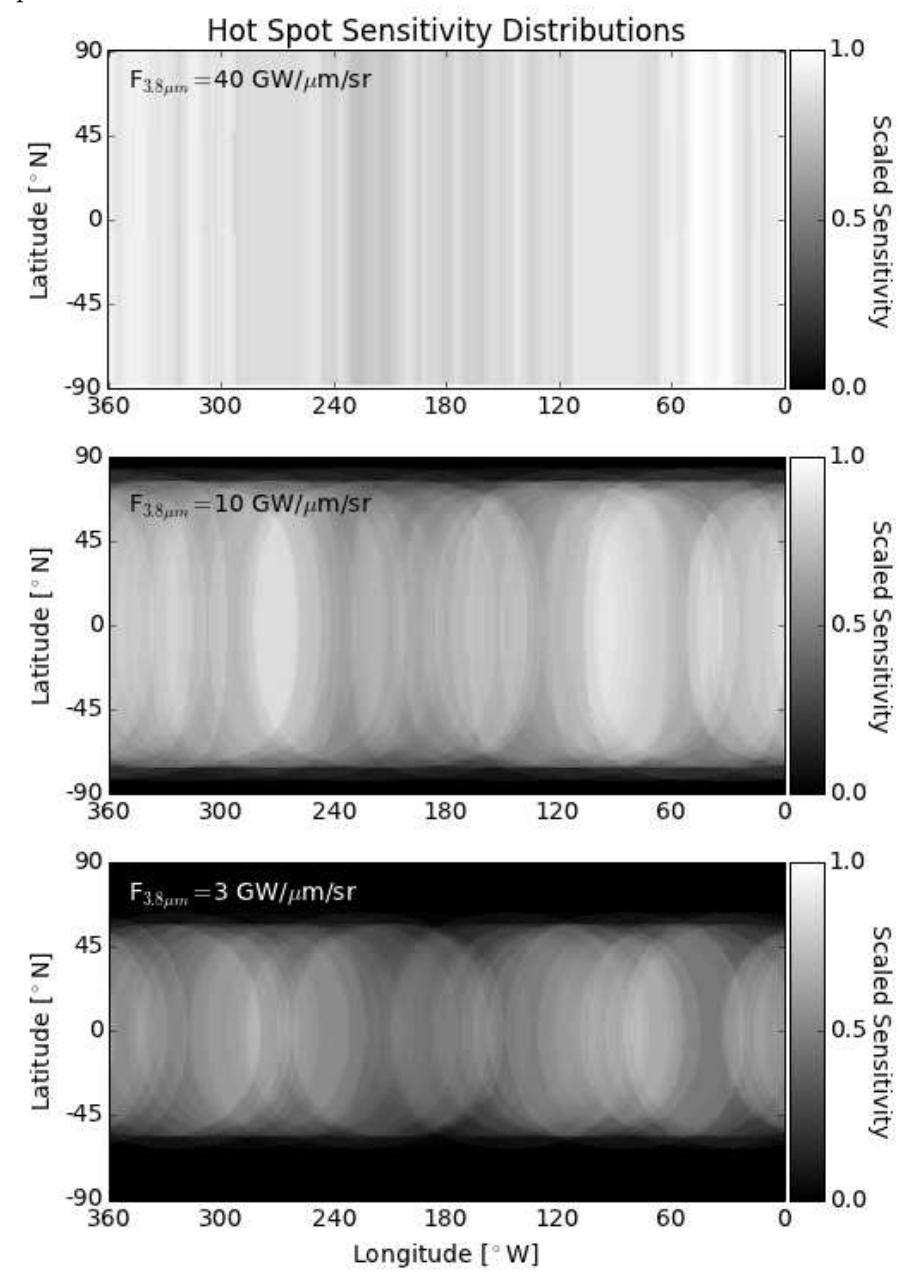

\subsection{Comparison to Galileo-era distribution 83 patera distribution}

Previous analyses of the spatial distribution of volcanism on Io have focused on the distribution of 4 paterae (Carr et al., 1998; Schenk et al., 2001; Radebaugh et al., 2001; Hamilton et al., 2013); of active 5 volcanism detected primarily by Galileo (Lopes-Gautier et al. 1999; Lopes et al. 2004); and of the heat 6 flow as estimated from Galileo observations (Veeder et al. 2012, 2015; Davies et al., 2015). We compare the longitudinal distribution of hot spots we detected with the distributions from previous work.

Like previous authors, we find an eastward offset in peak number density of hot spots of $30-60^{\circ}$ from the sub- and anti-jovian points. Our distribution is similar in overall character to the longitudinal distribution of 10 heat flow inferred from Galileo observations (Veeder et al., 2015). We note that this is not entirely expected, 11 since we are comparing number of hot spots with total heat flow, and it has been previously noted that 


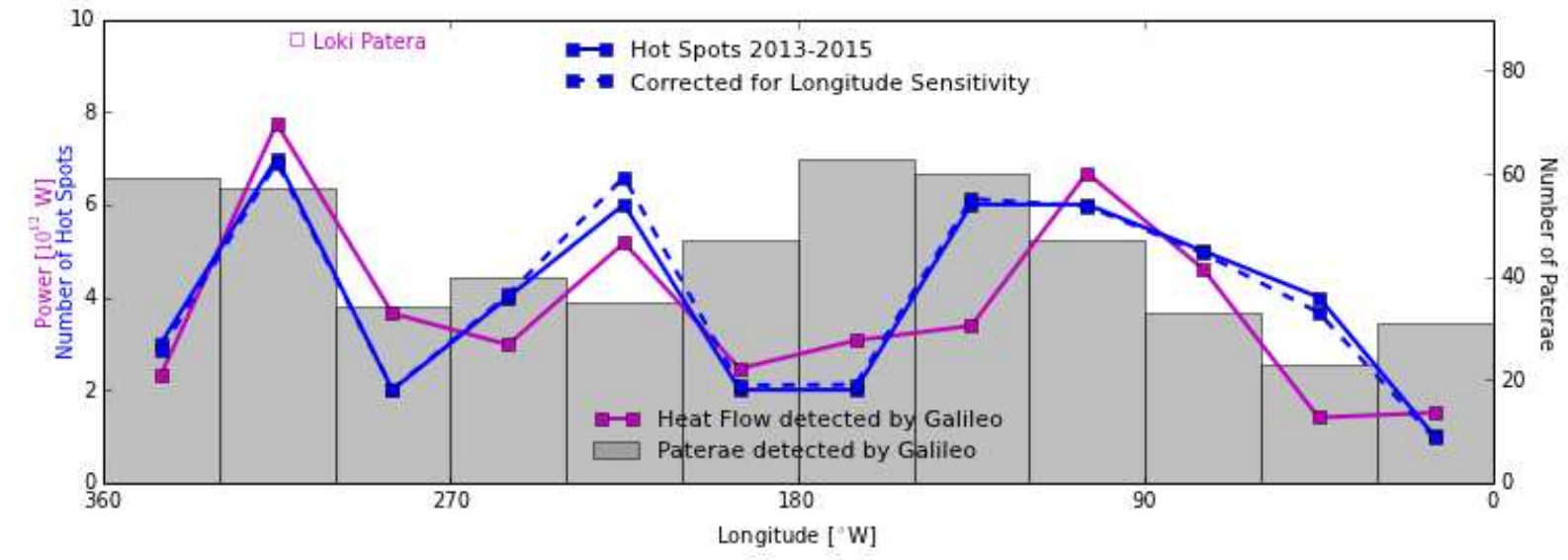

these properties are not closely correlated (Davies et al., 2015). However, since we are more likely to detect hot spots that are brighter and active for longer periods of time, we will preferentially detect the hot spots that contribute the most to Io's total heat flow, so that our distribution should indeed roughly mirror the distribution of total heat flow. In contrast, the distribution of paterae (e.g. Hamilton et al. 2013) does not contain information on the magnitude of heat flow from each location, and would not necessarily be expected to match the heat flow distribution. While the majority of the volcanic thermal emission measured from Galileo does come from paterae, at certain longitudes (e.g. $150^{\circ} \mathrm{W}-240^{\circ} \mathrm{W}$ ) a fair proportion of thermal emission comes from lava flow fields (Veeder et al. 2012; Figure 10).

Figure 8: Number of hot spots detected per $30^{\circ}$ longitude bin in 2013-2015, compared to distribution of heat flow detected by Galileo, and paterae. Galileo results are from Veeder et al. (2015) Fig. 11 (thermal emission), and exclude outburst eruptions. The total power of Loki Patera is shown separately, and would bring the total emission in its longitude bin to nearly 18 TW if included. Paterae numbers are from Hamilton et al. (2013) Fig. 3 for 529 bright, dark, and undivided patera floor units. The y-axis scale on the left side serves for both hot spot number in 2013-2015 and Galileo total power measurements. The mismatch between the 2013-2015 hot spot numbers and the Galileo power measurements (blue and purple lines) from 120 to $150^{\circ} \mathrm{W}$ may be due to the particularly high concentration of low-intensity hot spots in this region. be due to the particularly high concentration of low-intensity hot spots in this region.

The uniformity of our observations and roughly equivalent sensitivity to both the leading and trailing hemispheres allow us to confirm some previously-tentative conclusions. Veeder et al. (2012, 2015) and Davies et al. (2015) find that the region from $90^{\circ} \mathrm{W}$ to $180^{\circ} \mathrm{W}$ and $45^{\circ} \mathrm{S}$ to $45^{\circ} \mathrm{N}$ contains the greatest number of thermal sources, but note that this result may be biased by the high spatial resolution of Galileo observations of this region. Our dataset, which is in fact less sensitive to these longitudes than other regions, also finds a high concentration of thermal sources in this region, confirming the previous conclusion. This trend can be seen in the map in Figure 3. We note that it is less apparent in Figure 8 because this figure combines all latitudes, while the high hot spot concentration is focused at low to mid latitudes; in addition, the hot spots in this region are generally faint and contribute only minorly to the heat flow. 


\subsection{Comparison to Tidal Heating Models}

Previous studies have found greater heat flow from equatorial regions than polar regions, which was taken as evidence of an asthenospheric heating mechanism. Although we find that intense and rapidlyvarying eruptions occur primarily at mid to high latitudes, we estimate that the persistent hot spots of moderate intensity dominated Io's total heat flow during 2013-2015 (de Kleer and de Pater 2016), consistent with previous findings. However, solid-body asthenospheric heating models predict heat flow maxima at $90^{\circ} \mathrm{W}$ and $270^{\circ} \mathrm{W}$, while observations find these maxima offset.

In Figure 9, we compare the locations of detected hot spots with the predictions of tidal heating models for the cases of deep mantle heating and combination heating with a partial fluid layer, from Hamilton et al. (2013; based on model by Segatz et al., 1988) and Tyler et al. (2015) respectively. We find that hot spots in the leading hemisphere (longitude $<180^{\circ}$ ), and in particular the hot spots with persistent activity, fit well with the Tyler et al. (2015) model shown. The distribution matches less well in the trailing hemisphere, where nearly all large eruptions fall at locations of low predicted heat flow, and no hot spots at all were detected in the region of maximum predicted heat flow. In contrast, the large eruptions do correlate well with the distribution expected for deep mantle heating (Figure 9a), although no eruptions were detected at the highest latitudes, which are the most probable in the pure deep-mantle heating case.

The distribution of dark-floored paterae observed by Galileo follow a similar hemispheric asymmetry, where the locations of such paterae match the combination model for longitudes $<180^{\circ} \mathrm{W}$, while the region of maximum predicted heat flow is nearly lacking in dark paterae for longitudes $>180^{\circ} \mathrm{W}$ (Veeder et al., 2012; Tyler et al., 2015).

\subsection{Bright Southern-Latitude Eruptions: Implications}

We find that nearly all bright eruptions during $2013-2015$ occurred at latitudes between $40^{\circ} \mathrm{S}$ and $65^{\circ} \mathrm{S}$, and demonstrate in Section 4 that such an occurrence is unlikely at the $95 \%$ level if such eruptions occur randomly, even considering the small number of bright eruptions observed. In the previous section, we discussed the latitudinal distribution in terms of tidal heating models, where violent high-latitude eruptions are predicted by deep-mantle heating scenarios.

An additional consideration is the possibility that the bright eruptions in the southern hemisphere were triggered by earlier eruptions. Following the eruptions at Heno and Rarog Paterae in August 2013, the southern-latitude eruptions occurred in a monotonic eastward progression over the two years of observation; this can be seen clearly in the animation included in the Supplementary Material. This steady progression, combined with the low probability of this collection of eruptions occurring coincidentally, suggests the possibility of a physical link between the events. Bright eruptions on Io have occurred closely spaced in time in 

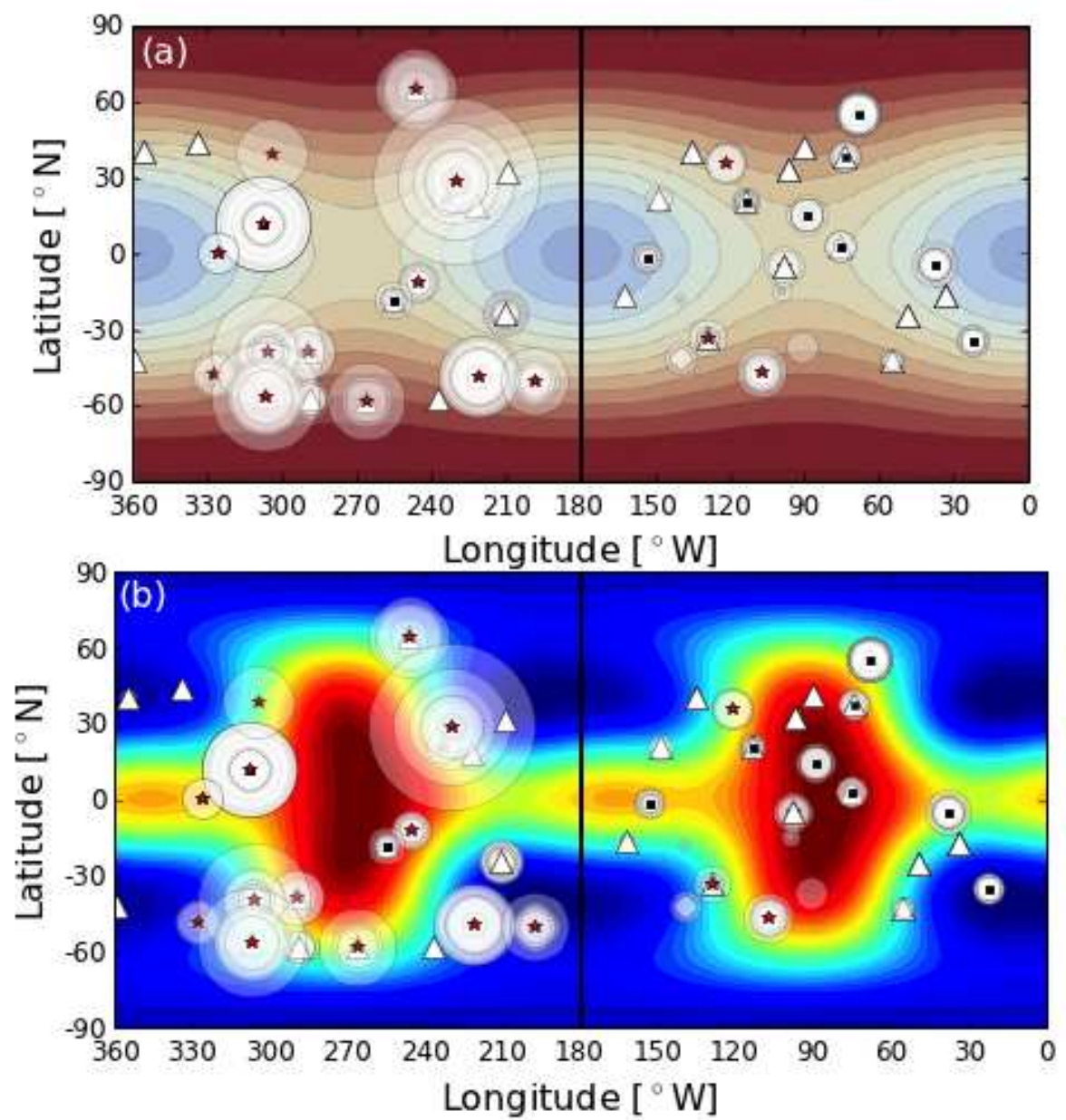

Figure 9: Locations of hot spot detections over-plotted on tidal heating model end-members in the case of (a) deep-mantle heating, base image from Hamilton et al. (2013) based on model by Segatz et al. (1988); and (b) combination heating with a partially-fluid interior, base image from Tyler et al. (2015). In both cases, red indicates regions of high heat flow, and blue regions of low heat flow. Black squares indicate persistent hot spots, and red stars indicate hot spots that exhibited bright eruption events. Low-intensity hot spots at longitudes $<180^{\circ} \mathrm{W}$ fit well with model (b), while bright hot eruptions at longitudes $>180^{\circ} \mathrm{W}$ do not fit with this model, and instead fit better with a deep-mantle heating model (a), despite the lack of detection of any very high latitude eruptions.

1 the past, as discussed in de Kleer and de Pater (2016).

There is evidence that some eruptions on Earth are triggered by large earthquakes up to a thousand $\mathrm{km}$ or more away (Linde and Sacks, 1998; Manga and Brodsky, 2006), or by other volcanic eruptions up to 200 $\mathrm{km}$ distant. The triggered eruptions can be delayed from the triggering event by as much as several months (Schminke 2004; Bautista et al., 1996). Some authors have argued that eruptions can be triggered at a 6 distance of $10^{3} \mathrm{~km}$ with a time lag of years in the case of viscoelastic relaxation of stresses as the triggering mechanisms (Hill et al., 2002; Marzocchi et al., 2004). Eruptions can be triggered due to external factors such as seismicity, or by internal factors such as large-scale magma movement (Manga and Brodsky, 2006). 9 Although these conclusions are considered tentative and are based primarily on temporal correlations, it is 
intriguing that the same sort of correlation is seen in this string of southern volcanoes on Io, which are each separated from the next by $\sim 500 \mathrm{~km}$. If such an association could be confirmed by future observations of temporally-correlated eruptions, the distances and timescales over which such triggering occurs would yield new insight into the triggering mechanisms and magma supply for Io's eruptions.

Regardless of whether the eruptions around $45-60^{\circ} \mathrm{S}$ were mutually triggered, their occurrence requires a large magma supply and violent eruption events to push through the lithosphere, consistent with a deep mantle magma source for these events.

\section{Conclusions}

We present an analysis of the spatial distribution of volcanic activity on Io based on 409 detections of 48 hot spots in 2013-2015; this analysis follows on a companion paper (de Kleer and de Pater 2016) which presents the data and timelines of thermal emission variability. In addition, we present here near-infrared maps of Io in numerous filters, during three separate epochs of observation. Based on our analysis, we conclude the following:

1. Clustering of large eruptions: We detected 9 hot spots that reached an L'-band brightness above $30 \mathrm{GW} / \mu \mathrm{m} / \mathrm{sr}$. These events occur exclusively on the trailing hemisphere, and primarily at latitudes of $40-65^{\circ}$. In a statistical analysis based on simulated data corrected for observational biases, we find that bright eruptions in our dataset are more clustered and occur at higher latitudes than in $\sim 95 \%$ of simulations which assume that eruptions occur at random locations. The eruptions between 45 and $60^{\circ} \mathrm{S}$ occurred in an eastward progression over the two years of observation, and we suggest the possibility that the earlier eruptions triggered the subsequent ones, perhaps beginning with the outbursts at Rarog and Heno Paterae in August 2013. However, we note that the most powerful eruption we detected (201308C) does not appear to have any nearby major events.

2. Location vs. eruption class: We find a substantial difference in the locations of hot spots that exhibit bright eruptions compared with hot spots that exhibit moderate, consistent activity. Persistent hot spots occur primarily between $\pm 30^{\circ} \mathrm{N}$ and on the leading hemisphere, while bright eruptions occur primarily between $40-65^{\circ} \mathrm{N}$ and S and on the trailing hemisphere. In de Kleer and de Pater (2016), we identify a class of "mini-outbursts", bright hot spots which exhibit temporal characteristics of outbursts but are a factor of $\sim 5$ less bright. In this analysis, we see that outbursts and "mini-outbursts" also follow similar spatial distributions, supporting the possibility that these smaller events are either outbursts captured a few days after their peak, or smaller-scale versions of the same type of eruption. 
3. Comparison to past activity: The longitudinal distribution of hot spot numbers observed in 20132015 is a close match to the longitudinal distribution of heat flow as observed by Galileo (Veeder et al., 2012). In particular, our data indicate that the peak in hot spot number is shifted eastward by $30-60^{\circ}$ from the sub- and anti-jovian points, in agreement with past findings. This match confirms that two years of ground-based observations of Io are indeed sufficient to recover some of the general properties of Io's heat flow distribution, and that this distribution does not vary significantly over decadal timescales.

4. Implications for tidal heating: The spatial distribution of hot spots detected in our dataset does not match the distributions predicted by solid-body asthenospheric or deep-mantle heating. While the hot spots on the leading hemisphere, and persistent hot spots everywhere on Io, match the predictions of recent models incorporating a partial fluid interior (Tyler et al., 2015), this model is a poor match to the distribution of eruptions on the trailing hemisphere, and of bright eruptions in particular. It may be that future developments in tidal heating models will improve this discrepancy. However, the distribution may also reflect inhomogeneities in the degree of mantle convection or lithospheric thickness, as was recently suggested by Davies et al. (2015).

While these observations give new insight into the distribution of thermal activity on Io, many of the unresolved questions could be addressed by future ground-based observations or by a dedicated Io mission. If the string of southern eruptions are indeed physically interconnected, future observations in the months following outburst eruptions may reveal similar connections. This dataset also reveals that bright eruptions which still fall well short of outburst intensity (i.e. 3.8- $\mu \mathrm{m}$ intensities of 30-80 GW/ $\mu \mathrm{m} / \mathrm{sr}$, compared with $>150 \mathrm{GW} / \mu \mathrm{m} / \mathrm{sr}$ for outburst eruptions) are more similar to outbursts than to persistent hot spots in terms of temporal characteristics and spatial distribution. These may be a viable avenue for studying high-energy eruptions on Io, and they occur much more frequently than outbursts. Future detections of new hot spots in previously-inactive regions could also point to evolution in the location of magma supply to the surface, or the opening of new heat pipes through the lithosphere.

This dataset demonstrates the value of high-cadence ground-based observations of Io. Frequent, highresolution images provide a statistically significant database of hot spot detections. A continued campaign of this nature would improve constraints on the spatial distribution of activity, as well as revealing any variability in the locations of eruptions of different classes. In addition, the possibility of eruption triggering is intriguing, but requires consistent observation to assert that large eruptions occur near other large eruptions a meaningful fraction of the time. Ideally, future programs of this nature will observe at multiple nearinfrared wavelengths spanning $2-5 \mu \mathrm{m}$, so that the distribution of eruption powers and temperatures can 
1 also be investigated.

\section{Acknowledgments}

The authors would like to thank A. Davies for valuable insight at many stages of this work; A. Davies and an anonymous reviewer for careful reading and thoughtful comments; and J. Rathbun for interesting and helpful discussion throughout. In addition, the authors are grateful to A. Stephens and T. Geballe for supporting the Gemini N observing program, and to the Gemini N observers for collecting the data. This research was partially supported by the National Science Foundation grant AST-1313485 to UC Berkeley and by the National Science Foundation Graduate Research Fellowship to K. de Kleer under Grant DGE-1106400. This work made use of the JPL Solar System Dynamics high-precision ephemerides through the HORIZONS system. Data were obtained with the W.M. Keck Observatory, which is operated by the California Institute of Technology, the University of California, and the National Aeronautics and Space Administration. The Observatory was made possible by the generous financial support of the W.M. Keck Foundation. The authors extend special thanks to those of Hawaiian ancestry on whose sacred mountain we are privileged to be guests. Without their generous hospitality, none of the observations presented would have been possible. 
References

Bautista, B.C., Leonila, B.B., Stein, R.R., et al., 1996. Relationships of regional and local structures in Mount Pinatubo activity. In Fire and Mud, ed. C Newhall, RS Punongbayan. Seattle: Univ. Wash. Press.

Carr, M.H., McEwen, A.S., Howard, K.A., Chuang, F.C., et al., 1998. Mountains and calderas on Io: Possible implications for lithosphere structure and magma generation. Icarus 135, 146-165.

Davies, A.G., Veeder, G.J., Matson, D.L., Johnson, T.V., 2015. Map of Io's volcanic heat flow. Icarus $262,67-78$

de Kleer, K., de Pater, I., Davies, A.G., Ádámkovics, M., 2014. Near-infrared monitoring of Io and detection of a violent outburst on 29 August 2013. Icarus 242, 352-364.

de Kleer, K., de Pater, I., 2016. Time variability of Io's volcanic activity from near-IR adaptive optics observations on 100 nights in 2013-2015.

de Pater, I., Davies, A.G., Ádámkovics, M., Ciardi, D.R., 2014. Two new, rare, high-effusion outburst eruptions at Rarog and Heno Paterae on Io. Icarus 242, 365-378.

Geissler, P., McEwen, A., Phillips, C., et al., 2004. Surface changes on Io during the Galileo mission. Icarus 169, 29-64.

Hamilton, C.W. et al., 2013. Spatial distribution of volcanoes on Io: Implications for tidal heating and magma ascent. Earth Planet Sci Lett 361, 272-286.

Hill, D.P., Pollitz, F., Newhall, C., 2002. Earthquake-volcano interactions. Phys Today 55, 41-47.

Hom, E.F.Y., Marchis, F., Lee, T.K., et al., 2007. AIDA: an adaptive image deconvolution algorithm with application to multi-frame and three-dimensional data. J Opt Soc Am A, 24, 1580-1600.

Khurana, K. et al., 2011. Evidence of a global magma ocean in Io's interior. Science 332, 1186-1189.

Laver, C. and de Pater, I., 2008. Spatially resolved $\mathrm{SO}_{2}$ ice on Io, observed in the near IR. Icarus 195, $752-757$.

Linde, A.T., Sacks, I.S., 1998. Triggering of volcanic eruptions. Nature 395, 888-890. 
Lopes, R.M.C., Kamp, L.W., Smythe, W.D., Radebaugh, J., Turtle, E., Perry, J., Bruno, B., 2004. Global distribution of active volcanism on Io as known at the end of the Galileo mission. LPSC XXXV, 1837.

Lopes-Gautier, R. et al., 1999. Active volcanism on Io: Global distribution and variations in activity. Icarus 140, 243-264.

Manga, M., Brodsky, E., 2006. Seismic triggering of eruptions in the far field: Volcanoes and geysers. Ann Rev Earth Planet Sci 34, 263-91.

Marzocchi, W., Zaccarelli, L., Boschi, E., 2004. Phenomenological evidence in favor of a remote seismic coupling for large volcanic eruptions. Geophys Res Lett 31, L046041.

Radebaugh, J. et al., 2001. Paterae on Io: A new type of volcanic caldera? J Geophys Res 106, 33005-33020.

Ross, M.N. et al., 1990. Internal structure of Io and the global distribution of its topography. Icarus 85, 309-325.

Schenk, P., Hargitai, H., Wilson, R., McEwen, A., Thomas, P., 2001. The mountains of Io: Global and geological perspectives from Voyager and Galileo. GJR 106, 33201-33222.

Schminke, H-U., 2004. Volcanism. Berlin/Heidelberg: Springer-Verlag. 324 pp.

Segatz, M. et al., 1988. Tidal dissipation, surface heat flow, and figure of viscoelastic models of Io. Icarus $75,187-206$.

Tyler, R.H., Henning, W.G., Hamilton, C.W., 2015. Tidal heating in a magma ocean within Jupiter's moon Io. ApJ Supp. 218-222.

Veeder, G.J., et al., 2009. Dark flow fields on Io. Icarus 204, 239-253.

Veeder, G.J., et al., 2011. Io: Heat flow from dark paterae. Icarus 212, 236-261.

Veeder, G.J., Davies, A.G., Matson, D.L., Johnson, T.V., Williams, D.A. and Radebaugh, J., 2012. Io: Volcanic thermal sources and global heat flow. Icarus 219, 701-722.

Veeder, G.J., et al., 2015. Io: Heat flow from small volcanic features. Icarus 245, 379-410.

Williams, D.A., Keszthelyi, L.P., Crown, D.A., 2011. Volcanism on Io: New insights from global geologic mapping. Icarus 214, 91-112. 